\title{
Inference of Pure Titanium Micro-Arc Oxidation Process on Microstructure of Calcium Phosphate Membrane Layer
}

$$
\begin{gathered}
\text { Yu-lei LI }{ }^{\mathrm{a}, \star} \text {, Jin-jun TANG } \\
\text { Xiao-yu } \text { JIN }^{\mathrm{e}}
\end{gathered}
$$

Ningbo Sub-academy of the National Weapons Science Research Academy, Ningbo 315103, China

aliyulei7395@163.com, bjinjun.tang@163.com, 'daiyuelai_1988@126.com, zhanglijun_nb@126.com, jinxiaoyu_nb@163.com

${ }^{*}$ Corresponding author

Keywords: Pure titanium, Micro-arc oxidation, Calcium phosphate membrane layer, microstructure.

\begin{abstract}
In this paper, the growth law of the phosphate and calcium layer in different voltage process was studied. The surface morphology of the film was observed by SEM, and then the distribution of phosphorus and calcium in the film was analyzed. The results show that the higher the voltage, the more uniform the pore size in the film layer, the larger the pore size, the lower the pore density and the lower porosity. And with the increase of voltage, the content of calcium and phosphorus in the film layer increased, the ratio of calcium and phosphorus content remained basically unchanged.
\end{abstract}

\section{Introduction}

Micro-arc oxidation technology is a chemical, electrochemical and plasma chemistry interaction process. Reaction process contains a high voltage spark discharge in electrolyte aqueous solution process, it can generate high performance on metal surface, strong ceramic oxide layer ${ }^{[1]}$ through thermal chemical reaction. Elements can be modified in the form of dissolved salt exists in the electrolyte, thus through reaction into $^{[2]}$ the modified membrane layer, is a simple, economic and environmentally friendly preparation of metal ceramic oxide coating on the surface of the technology, which has become a research hotspot of the metal material surface modification technology at home and abroad ${ }^{[3-5]}$.Pure titanium plate as the research object, through the pretreatment technology research, micro-arc oxidation surface modification technology research, and combined with the performance test, analyzing the effect of calcium phosphate membrane layer of different process parameters on the micro-arc oxidation, which provides the basis of the research for medical pure titanium micro-arc oxidation film layer.

\section{Experiment}

Purchase of TA2 pure titanium plate, material chemical composition and content as shown in table 1 .Wire-cutting processing is $15 \mathrm{~mm} * 15 \mathrm{~mm} * 3 \mathrm{~mm}$ sample. Burnishing the sample in 400 \#, 800 \#, 1200 \# water sand paper in turn to remove the black oxide. Sample after polishing with acetone, ethanol, deionized water, in turn, ultrasonic washing $15 \mathrm{~min}$, removing grease and residual impurity particles attachments, drying and setting aside. Ultrasonic cleaning process is shown in Fig.1. This is adopted 
in the experiment of Harbin industrial university's centre for HeiLongJiang science and technology cooperation between China and Russia and industrialization production WHD - 20 micro-arc oxidation equipment, hanging the sample to the central as anode, electrolyte and electrode place making certain insulation processing, stainless steel water tank as cathode, after setting up the electric parameters, connected to the power supply. The micro-arc oxidation process is shown in Fig.2. After the micro-arc oxidation, turning the power off, removing samples, deionizing water to clean, drying and setting aside. Electrolytic liquid : calcium hydroxide $1.2 \mathrm{~g} / \mathrm{L}, 4 \mathrm{~g} / \mathrm{L}$ sodium hexametaphosphate, potassium fluoride $8 \mathrm{~g} / \mathrm{L} .320 \mathrm{v}, 400 \mathrm{v}$, oxidation time is $5 \mathrm{~min}$. Scanning electron microscopy observation of surface morphology, measuring element percentage.

Table 1. The atomic percentage of the other elements contained in the material

\begin{tabular}{|c|c|c|c|c|c|}
\hline composition & $\mathrm{Fe}$ & $\mathrm{C}$ & $\mathrm{N}$ & $\mathrm{H}$ & $\mathrm{O}$ \\
\hline Content (at\%) & 0.2 & 0.05 & 0.03 & 0.008 & 0.1 \\
\hline
\end{tabular}

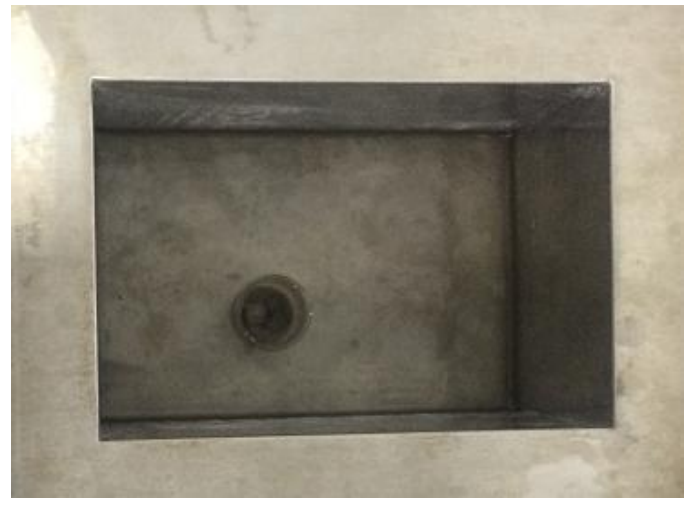

Fig.1.Ultrasonic cleaning

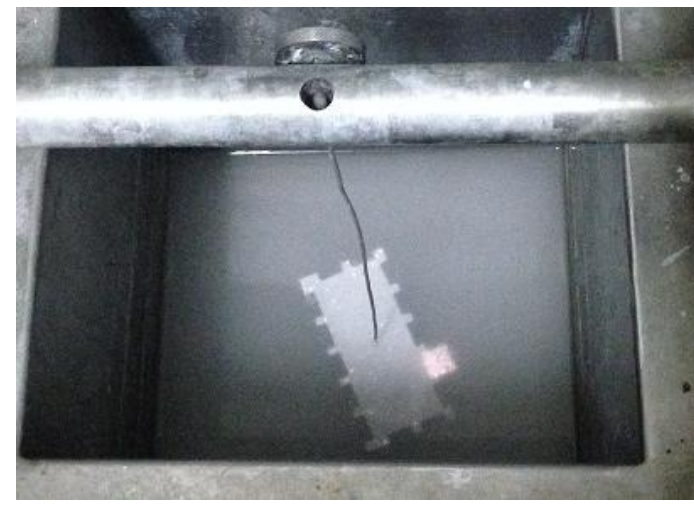

Fig.2.Micro-arc oxidation

\section{Results and discussion}

The ceramic membrane formation on the surface of pure titanium metal conform to the general law of micro-arc oxidation. On the surface of the high temperature melt after micro-arc discharge channel filling, plasma discharge reaction gradually stop. But a certain position after discharge sparks, another weak point in the film breakdown discharge will happen again, and then ejected the melt will cover discharge channel. So again and again,repeat the process of discharge, melting, spraying, cooling and solidification. Micro-arc oxidation film layer thickening of outward expansion, finally forms the microstructure observed in the SEM images similar to the porous morphology of the crater. So the micro-arc oxide film formation process is complex, the nature of the substrate, conductive ability of electrolyte, voltage, etc. As shown in figure 3, the surface morphology of the oxide film under different voltage processes is different. Voltage on $320 \mathrm{v}$, the number of holes was significantly more than $400 \mathrm{v}$. 
Table 2. Under different voltage process of micro-arc oxidation membrane layer of calcium phosphorus content and ratio

\begin{tabular}{|c|c|c|c|}
\hline element & $\mathrm{Ca}(\mathrm{at} \%)$ & $\mathrm{P}(\mathrm{at} \%)$ & $\mathrm{Ca} / \mathrm{P}$ \\
\hline $320 \mathrm{v}$ & & & \\
\hline $400 \mathrm{v}$ & 6.47 & 5.85 & 1.106 \\
\hline
\end{tabular}

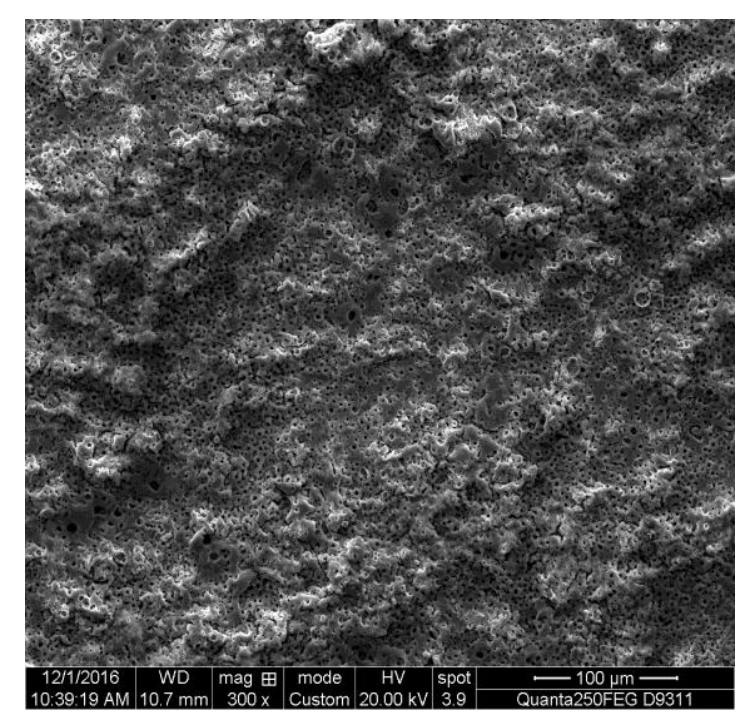

(a) $320 v, 300 x$

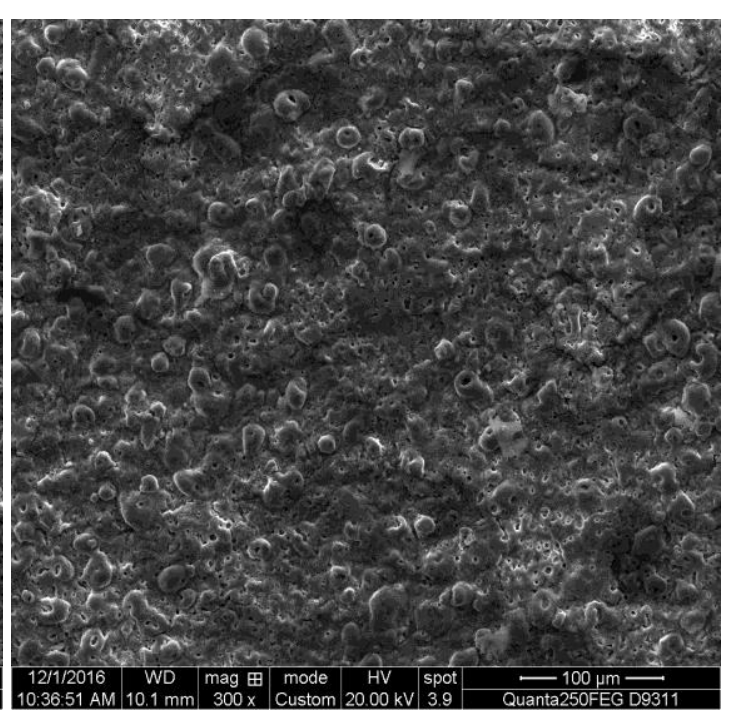

(b) $400 v, 300 x$

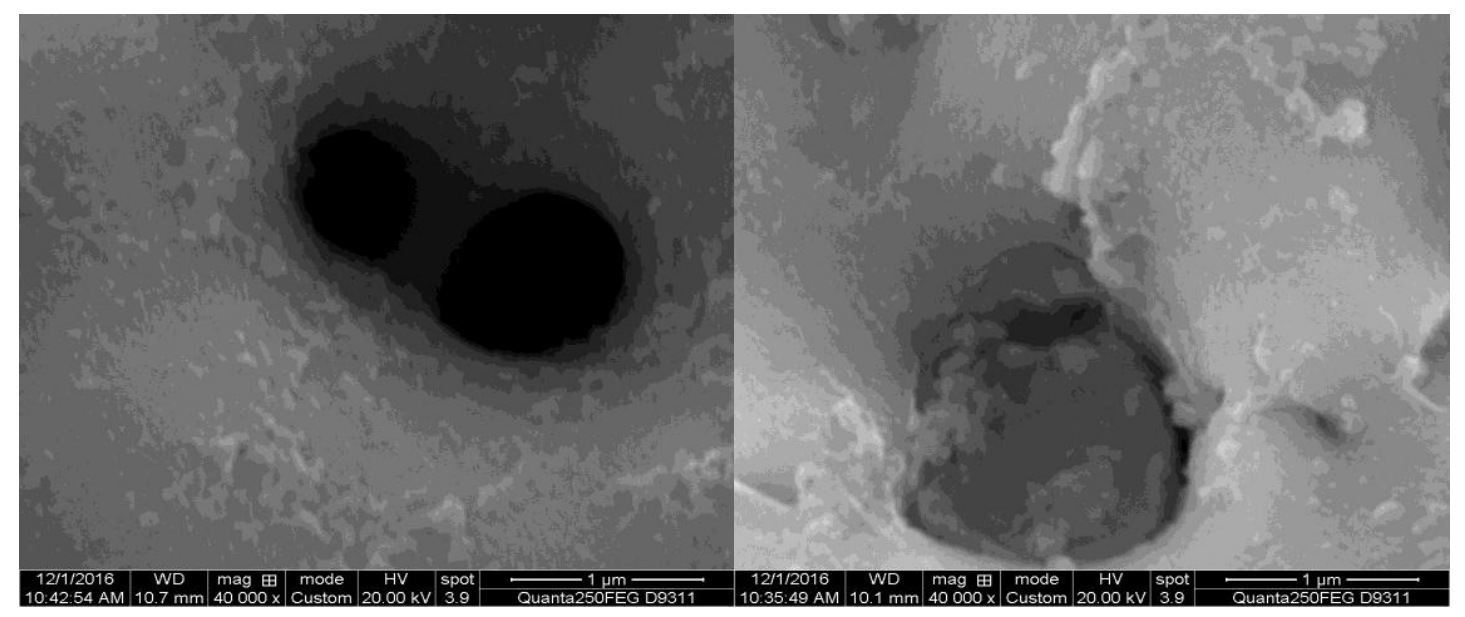

(c) $320 v, 40000 x$

(d)400v,40000x

Fig.3.The micro-arc oxidation film under different voltage process layer surface morphology

As shown in Fig.3 (a), under the condition of 320v voltage, there are a large number of large diameter holes similar to the groove in the film layer. In the process of voltage increase, the formation speed of the oxide film is accelerated, and the heat is released from the surface when the breakdown occurs. A part of the melt is ejected, and the heat released by the cooling and solidification increases the temperature of the electrolytic solution and promotes the corrosion of the oxide film. At this time the resistance decreases, the current increases, the oxide film growth rate increased again until the 
dissolution rate to reach equilibrium, the thickness increased to the voltage is difficult to breakdown, the hole around the weak location of the probability of repeated breakdown is reduced, easy to form uniform pore structure . Compared to (a) and (b) in Fig.3, it is not difficult to find that the hole size in (b) is more uniform than (a). Compared to (c) and (d) in Fig.3, the latter has a larger hole size than the former. But similar to Figure (c) of the hole arrangement, easy to breakdown ultimately form a groove structure.

With the higher voltage, number of holes is reduced, surface porosity decrease. When the voltage is low, groove structure is easy to form with two holes or porous communication. At this time the porosity is significantly higher than the pore structure. As the voltage increases, the groove structure disappears, more and more round holes appears, the higher the voltage, the larger the pore size, the lower the pore density and the porosity.

Under the action of strong electric field, the electrolyte ions through electrophoresis method into the channel, the pure titanium substrate contact with calcium phosphate salt electrolyte by plasma channel. The element of calcium and phosphorus can be successfully involved in the formation of ceramic film. As shown in Table 2, the content of calcium and phosphorus in the film with 400v parameter is higher, and the ratio of calcium and phosphorus remains basically unchanged. With the increase of voltage, the discharge channel will produce higher temperature and pressure, more electrolyte ions participate in the reaction, and finally make the content of calcium and phosphorus in the film increased.

\section{Conclusion}

Preparation of calcium phosphorous membrane layer,there is the phenomenon with the higher the voltage, the more uniform the hole size, the larger the aperture, the smaller the number of holes and the lower the porosity.

With the increase of voltage, the content of $\mathrm{Ca}$ and $\mathrm{P}$ in the film increased, and the ratio of $\mathrm{Ca}$ to $\mathrm{P}$ remained basically unchanged.

\section{References}

[1] Nyan M,Tsutsumi Y,Oya K,et a1. Synthesis of novel oxide layers on titanium by combination of sputter deposition and micro-arc oxidation techniques[J]. Dental Materials Journal,2011,30(5): 754-761.

[2] Zhao GL,Li X,Xia L,et a1. Structure and apatite induction of a microarc-oxidized coating on

a biomedical titanium alloy[J]. Applied Surface Science,2010,257(5): 1762-1768.

[3] Lee S H,Kim H W,Lee E J,Li L H. Hydroxyapatite-TiO2 Hybrid Coating on Ti Implants[J]. Journal of Biomaterial applications,2006,20(3):195-208.

[4] Liang B,Fujibayashi S,Neo M,Tamura J,Kim H M,Uchida M, Kokubo T. Histological and mechanical investigation of the bone-bonding ability of anddically oxidized titanium in rabbits[J]. Biomaterials,2003,24.

[5] Li L H,Kong $\mathrm{Y}$ M,Kim $\mathrm{H}$ W,Kim H E,Heo S J. Improved bioligical performance of $\mathrm{Ti}$ implants due to surface modification by micro-arc oxidation[J]. Biomaterials,2004,25: 2867-2875. 